\title{
Le comportement des alluvions du Rhône dans une grande boîte de cisaillement direct
}

\section{Y. BOURDEAU}

INSA de Lyon,

URGC Géotechnique, bât. 304

20, avenue Albert-Einstein, 69621 Villeurbanne Cedex
Cet article présente une procédure d'exploitation d'essais de cisaillement direct réalisés avec une grosse boite sur différents sites de la région lyonnaise ainsi qu'en laboratoire. Elle conduit à des lois empiriques de formulation simple pour déterminer les paramètres de cisaillement des alluvions du Rhône. La première partie décrit le mécanisme de l'évolution réciproque de l'angle de frottement interne et de la cohésion avec le déplacement imposé, en précisant l'influence des caractéristiques physiques et granulométriques sur le comportement observé. La deuxième partie décrit l'évolution conjointe de la dilatance avec la mobilisation de la résistance au cisaillement et les deux lois d'écoulement qui en résultent. Parmi les enseignements de cette étude phénoménologique on met en évidence le rôle temporaire de la cohésion capillaire dans les alluvions du Rhône et l'effet du remaniement sur les paramètres de cisaillement. On montre aussi que l'angle de frottement critique des alluvions peut être estimé avec un excès de l'ordre de un degré au point de soulèvement optimal dans un essai de cisaillement direct à disposition symétrique.

\section{The behaviour of Rhône alluvium materials in a large direct shear test}

This paper presents the approach for exploitation of large direct shear tests carried out in various sites for the Lyon area and also in laboratory. Empirical models for determination of shear parameters are given for Rhône alluvium materials. The first part of the study describes the variations of the internal friction angle and of the cohesion as a function of displacement. The influence of physical properties and particle size on this behaviour is specified. The second part describes the flow rules which may be used to express the relationship between the mobilized shear stress and the rate of dilation. This investigation shows the temporary effect of the cohesion and the effect of disturbance on shear parameters. It also appears that the critical state for alluviums can be estimated with an excess about one degree when zero rate of dilation occurs in a symmetrical test with fixed top platen. 


\section{Introduction}

Une superficie importante du site de Lyon est recouverte par des dépôts d'alluvions du Rhône, formés de sables et graviers, pouvant atteindre une vingtaine de mètres d'épaisseur. Le niveau de la nappe phréatique affleure le plus souvent le toit des alluvions, à la limite des remblais récents de 4 à $5 \mathrm{~m}$ d'épaisseur qui constituent la couverture naturelle en site urbain. Les terrassements ou l'exécution de fondations profondes mettent souvent en évidence des hétérogénéités dans cette couche porteuse, la présence d'horizons sableux alternant avec des dépôts plus grossiers (David, 1967). La figure 1 présente des courbes granulométriques relatives à une fouille de $5 \mathrm{~m}$ de profondeur, qui rendent compte de l'hétérogénéité de ces alluvions et de la taille des grains.

Ces sables et graviers sont de composition quartzique et contiennent des éléments calcaires qui leur confèrent une cohésion capillaire naturelle due à une cémentation des grains par des dépôts de carbonate de calcium (Cambefort, 1965). Des talus sablo-graveleux de grande hauteur peuvent ainsi rester stables avec une pente élevée, mais cet équilibre reste cependant fragile.

L'appréciation de cette cohésion en vue du dimensionnement des ouvrages est très délicate et source de nombreux débats. D'une manière plus générale, la détermination des paramètres de cisaillement des sols grossiers au laboratoire est sujette à l'écrêtage ou à la reconstitution du matériau, ce qui rend indispensable l'utilisation de matériels d'essais in situ de grandes dimensions pour caractériser ces alluvions.

Cet article présente la synthèse de résultats d'essais de cisaillement direct obtenus sur le sol en place, à Lyon et sur des sites voisins de la vallée du Rhône pré-

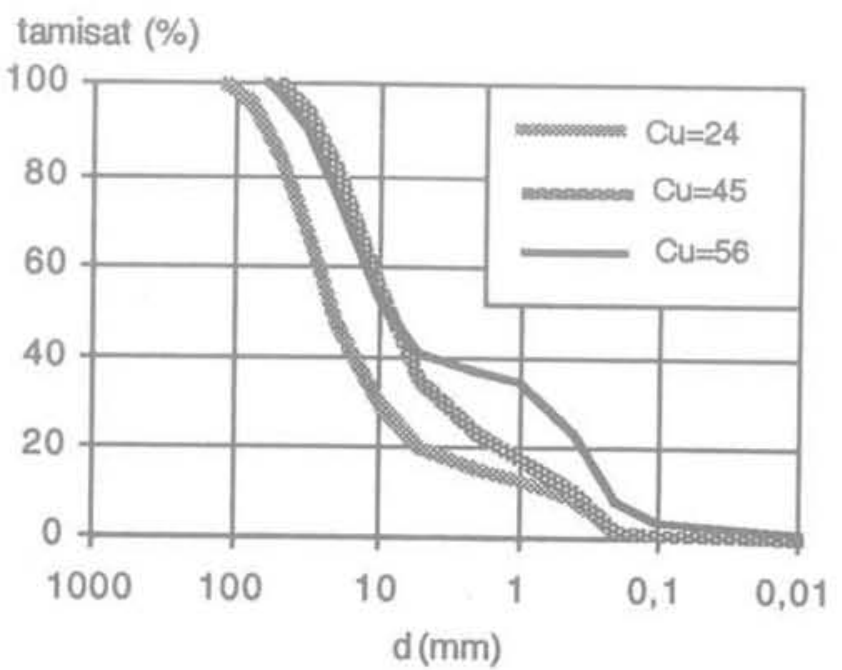

FG.1 Exemple de courbes granulométriques des alluvions du Rhône (gare de Vénissieux).

Example of grain size distribution for Rhōne alluvium materials (station of Vénissieux). sentant des alluvions semblables (Laréal et al., 1973, Laréal, 1975, Kastner, 1982, Bourdeau et al., 1989), ainsí qu'au laboratoire sur des échantillons reconstitués (Bacot, 1981, Guichard, 1995, Ogunro, 1996). Cette étude expérimentale, réalisée avec une boite de section $0,6 \mathrm{~m} \times 0,6 \mathrm{~m}$ et de hauteur de $0,4 \mathrm{~m}$, est complétée par la mesure de l'angle de frottement critique $\varnothing$ à l'appareil triaxial.

Le comportement au cisaillement des alluvions du Rhône est analysé ici à travers l'évolution réciproque de l'angle de frottement interne $\emptyset$ et de la cohésion $C$ jusqu'à l'obtention de l'effort résiduel. Ce mécanisme, qui est décrit conjointement au développement de la dilatance, débouche sur des lois d'écoulement.

Une approche par corrélations faisant intervenir les caractéristiques physiques, granulométriques et mécaniques du matériau conduit à des lois de comportement générales, qui sont de formulation simple et complémentaires. Elles englobent les essais in situ et les essais de laboratoire et permettent de répondre à l'effet de l'écrêtage et du remaniement sur la résistance au cisaillement des alluvions du Rhône.

\section{2}

\section{Les dispositifs expérimentaux}

\section{1}

\section{Les essais in situ}

Les expérimentations en place ayant été réalisées dans le cadre de grands travaux, parfois à l'intérieur de fouilles étroites en site urbain, le dispositif d'essais a été conçu pour répondre avec souplesse aux impératifs habituels des chantiers: conditions d'accès difficiles, encombrement limité, rapidité d'intervention pour le respect des plannings et de l'organisation du chantier et utilisation d'engins pour les manutentions.

La boite, qui ne comprend que la partie dite supérieure, est mise en place par havage sur une profondeur de $350 \mathrm{~mm}$. Pour empêcher toute possibilité d'évolution de la charge verticale en cours d'essai et pour autoriser la libre évolution de la dilatance dans l'échantillon, le dispositif de chargement est réduit à un plateau qui vient en appui sur l'échantillon de sol (Fig. 2a). Ce plateau peut recevoir jusqu'à 10 gueuses en fonte de $5 \mathrm{kN}$, ce qui limite la contrainte normale $\sigma$ à $158 \mathrm{kPa}$ en tenant compte du poids de la boîte et du matériau qu'elle contient.

Le système de traction comprend un capteur d'effort et un vérin actionné par une centrale hydraulique. L'effort de cisaillement est appliqué dans le plan de séparation du boîtier et du massif selon le processus du déplacement contrôlé à charge normale constante, avec une vitesse de $1 \mathrm{~mm} / \mathrm{mn}$. Le déplacement horizontal U est mesuré en deux points de la face arrière de la boîte et les mouvements verticaux $\mathrm{V}$ du plateau ont pu être suivis sur le site de Saint-Vallier.

\section{2}

\section{Les essais de laboratoire}

Dans le dispositif de laboratoire (Fig. 2b) la boite supérieure et les modes d'application de la charge ver- 
charge normale

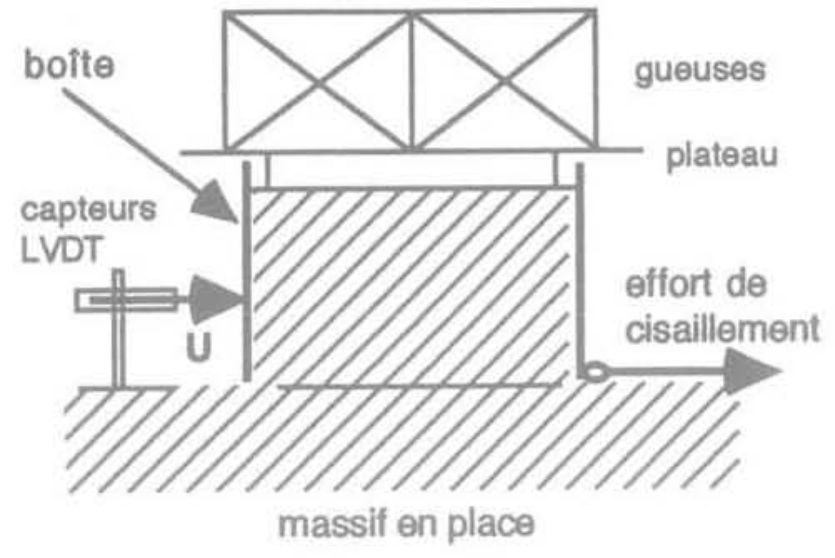

(a)

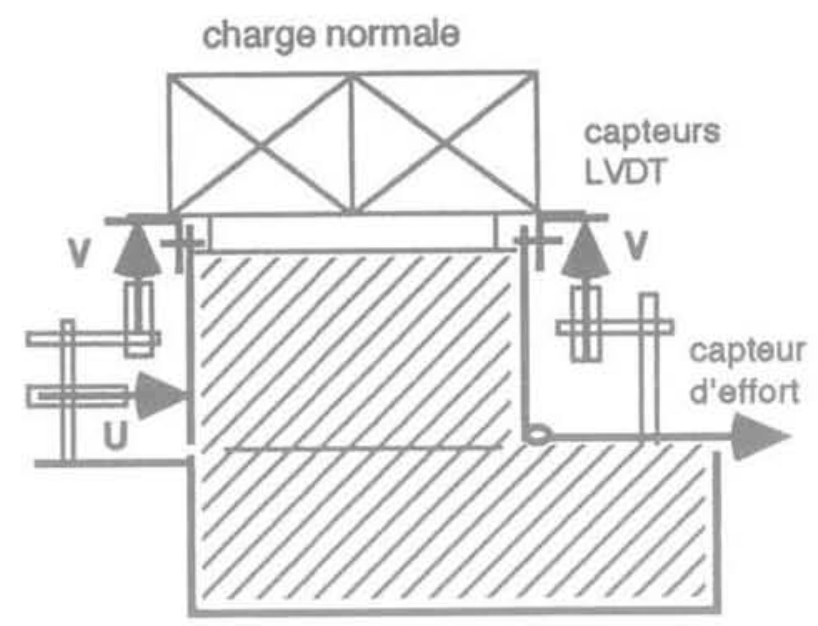

(b)

FG. 2 Principe de l'essai de cisaillement direct: (a) essai in situ avec plateau de chargement libre et boîte $0,6 \mathrm{x}$ $0,6 \mathrm{~m}$ (section) x 0,4 (hauteur), (b) essai au laboratoire avec piston bloqué (disposition symétrique).

Large direct shear test (schematic) : (a) in situ test with free top platen and box $0,6 \mathrm{~m} \times 0,6 \mathrm{~m}(\mathrm{area}) \times 0,4 \mathrm{~m}(\mathrm{height})$, (b) laboratory symmetrical test with fixed top platen.

ticale et de l'effort de cisaillement sont identiques à ceux de l'essai en place. La longueur de la boite inférieure est portée à $1 \mathrm{~m}$ pour avoir une section de cisaillement constante et un écartement suffisant est imposé entre les boîtiers afin d'éviter les frottements parasites.

Le mode opératoire s'effectue sans le souci de la reproduction des conditions relevées sur site et il est modifié pour chaque série d'essais afin d'étendre la plage des caractéristiques physiques et granulométriques du sol. Le compactage en plusieurs couches est assuré avec une dame à hauteur de chute contrôlée ou une pilonneuse.

Alors que les mouvements relatifs de la boîte et du plateau de chargement ne sont pas empêchés dans les essais in situ, la liberté de rotation de l'échantillon est réduite au laboratoire en appliquant la disposition symétrique recommandée par Jewell (1989) pour accroître l'uniformité des déformations dans le plan de cisaillement. Cette opération consiste à solidariser le plateau au boîtier supérieur, dont les faces intérieures sont lubrifiées, après chargement et consolidation du matériau.

Les mouvements verticaux $\mathrm{V}$ sont mesurés aux quatre coins du boîtier supérieur.

\section{3}

\section{Le programme expérimental}

Le bilan de la campagne expérimentale s'établit à 27 séries d'essais, pour $\sigma_{0}$ variant entre 36 et $158 \mathrm{kPa}$. Les essais in situ (Tableauli) ont été réalisés au toit des alluvions, après décapage de la couche superficielle, pour les sites de Saint-Vallier, Vénissieux, Feyzin, Millery, ou à l'intérieur de fouilles pour les sites Lyonnais (Part-Dieu, Saxe-Gambetta et Garibaldi) à des profondeurs pouvant atteindre $11 \mathrm{~m}$ par rapport au terrain naturel, ou $7 \mathrm{~m}$ par rapport au toit des alluvions. En outre, à Saint-Vallier, des essais complémentaires ont été réalisés sur des remblais reconstitués avec le matériau en place.
Les essais de laboratoire sont effectués sur des échantillons écrêtés à $\mathrm{d}_{\max }=80 \mathrm{~mm}, 50 \mathrm{~mm}, 31,5 \mathrm{~mm}$ ou $20 \mathrm{~mm}$ (Tableau II). Afin de définir la valeur minimale de $\mathrm{d}_{\text {mix }}$ permettant de conserver le comportement en place du matériau, l'écrêtage est réalisé sans compensation.

Le facteur d'amélioration $\mathrm{F}_{\mathrm{a}}$ indiqué aux tableauxI et II traduit l'influence conjuguée du poids volumique sec $\gamma_{d}$ et du coefficient d'uniformité Cu du matériau sur l'amélioration de la résistance au cisaillement. La relation empirique (2) qui détermine $F_{\text {. }}$ est une généralisation de la formule (1) proposée par Mogami (1969) pour estimer l'angle de frottement au pic $\varnothing$ des matériaux grossiers perméables à partir de l'indice des vides e et de Cu.

$$
(1+e) \sin \varnothing_{\text {pic }}=k=A+B \cdot \log (\mathrm{Cu})
$$

Les constantes $A$ et $B$ dépendent du sol. La solution $\mathrm{A}=2 \mathrm{~B}=0,432$ retenue dans (2) correspond pour les alluvions du Rhône à la borne supérieure des points de mesure dans le repère $\mathrm{k}-\log (\mathrm{Cu})$ (Bourdeau et al., 1989 et Guichard, 1995).

$$
\mathrm{F}_{\mathrm{n}}=\frac{0,216 \log (100 \mathrm{Cu})}{1+e}
$$

\section{4}

\section{Résultats expérimentaux}

\section{1}

\section{Estimation de l'angle de frottement critique}

Le bas du tableau II rend compte des essais triaxiaux avec mesure des variations de volume réalisés pour plusieurs densités d'un échantillon de diamètre $200 \mathrm{~mm}$ et de hauteur $420 \mathrm{~mm}$. L'angle de frottement critique $\varnothing$ dépendant essentiellement de la nature minéralogique du matériau, afin de réduire les effets d'échelle d'une part et pour ne pas avoir à tenir compte d'une cohésion capillaire d'autre part, ces essais triaxiaux ont été conduits sur un échantillon sec écrêté à $5 \mathrm{~mm}$ et débarassé des fines. 
TABLEAUI Programme des essais in situ. Set of in situ tests.

\begin{tabular}{|c|c|c|c|}
\hline Site & $\begin{array}{c}\gamma \mathrm{d} \\
\mathrm{kN} / \mathrm{m}^{3}\end{array}$ & $\mathrm{Ca}$ & $F_{i}$ \\
\hline $\begin{array}{l}\text { Lyon Part-Dieu (4 séries) } \\
\text { (TN à NGF } 167,6 \text { toit } \\
\text { des alluvions à NGF 163,2) } \\
\text { fouille à NGF } 160,5 \text { (I) } \\
\text { fouille à NGF } 158,5 \text { (II)" } \\
\text { fouille à NGF } 158,7 \text { (III)" } \\
\text { fouille à NGF } 156,8 \text { (IV)" } \\
\text { 'essais stoppés au pic } \\
\text { "valeur moyenne du site }\end{array}$ & $21.2^{B}$ & $60^{\mathrm{b}}$ & 0,653 \\
\hline $\begin{array}{l}\text { Lyon Saxe-Gambetta } \\
\text { (6 sêries) (TN à NGF } \\
167,2 \text { toit à NGF } 162,3 \text { ) } \\
\text { fouille à NGF } 159,6 \text { ( } 2 \text { séries) } \\
\text { fouille à NGF } 158 \text { (2 séries) } \\
\text { fouille à NGF } 156 \text { (2 séries) }\end{array}$ & $\begin{array}{l}17 \\
19,4 \\
21,1\end{array}$ & $\begin{array}{r}243 \\
37 \\
60\end{array}$ & $\begin{array}{l}0,608 \\
0,564 \\
0,650\end{array}$ \\
\hline \multicolumn{4}{|l|}{$\begin{array}{l}\text { Lyon Garibaldi } \\
(T N \text { à NGF } 171 \text { toit } \\
\text { des alluvions à NGF 166) } \\
\text { fouille NGF 164.6 (1 série) }\end{array}$} \\
\hline $\begin{array}{l}\text { Vênissieux } \\
\text { toit des alluvions (1 série) }\end{array}$ & 18,5 & 56 & 0,584 \\
\hline \multicolumn{4}{|l|}{$\begin{array}{l}\text { Feyzin } \\
\text { toit des alluvions (1 série) }\end{array}$} \\
\hline $\begin{array}{l}\text { Millery } \\
\text { toit des alluvions (1 série) }\end{array}$ & 22,4 & 97 & 0.728 \\
\hline $\begin{array}{l}\text { Saint-Vallier ( } 5 \text { séries) } \\
\text { toit des alluvions (I) } \\
\text { toit des alluvions (II) } \\
\text { remblai roulé (III, } 2 \text { séries) } \\
\text { remblal déversé (IV) } \\
\text { "mesure de la dilatance }\end{array}$ & $\begin{array}{l}22,1 \\
21,1 \\
22,0 \\
21,1\end{array}$ & $\begin{array}{l}46 \\
60 \\
48 \\
40\end{array}$ & $\begin{array}{l}0,660 \\
0,650 \\
0,660 \\
0,620\end{array}$ \\
\hline
\end{tabular}

TABLEAUII Programme des essais de laboratoire. Set of laboratory test.

\begin{tabular}{|c|c|c|c|}
\hline Lieu de prélèvement & $\begin{array}{c}\gamma \mathrm{d} \\
\mathrm{kN} / \mathrm{m}^{4}\end{array}$ & $\mathrm{Cu}$ & $\mathrm{F}_{\text {a }}$ \\
\hline $\begin{aligned} \text { Miribel (6 séries) } \\
\mathrm{d}_{\max }=80 \mathrm{~mm} \\
\mathrm{~d}_{\max }=50 \mathrm{~mm}^{\mathrm{d}} \\
\mathrm{d}_{\max }=31,5 \mathrm{~mm}\left(\mathrm{I}^{\mathrm{d}}\right. \\
\mathrm{d}_{\max }=31,5 \mathrm{~mm} \text { (II) } \\
\mathrm{d}_{\max }=20 \mathrm{~mm} \text { (I) } \\
\mathrm{d}_{\max }=20 \mathrm{~mm} \text { (II) } \\
d_{\text {mesure de la dilatance }}\end{aligned}$ & $\begin{array}{l}20,9 \\
22,0 \\
24,4 \\
21,0 \\
21,4 \\
21,4\end{array}$ & $\begin{array}{l}64 \\
51 \\
37 \\
33 \\
22 \\
31\end{array}$ & $\begin{array}{l}0,648 \\
0,665 \\
0,710 \\
0,602 \\
0,583 \\
0,606\end{array}$ \\
\hline $\begin{array}{l}\text { Vénissieux (1 série) } \\
\mathrm{d}_{\max }=31,5 \mathrm{~mm}\end{array}$ & 20,2 & 36 & 0,566 \\
\hline $\begin{array}{l}\text { Satolas (1 série) } \\
\mathrm{d}_{\max }=20 \mathrm{~mm}\end{array}$ & 20,9 & 18 & 0,555 \\
\hline $\begin{array}{l}\text { Miribel ( } 3 \text { sẻries de } \\
\begin{array}{l}4 \text { essais triaxiaux }) \\
d_{\max }=5 \mathrm{~mm} \\
d=5 \mathrm{~mm} \\
d_{\max }=5 \mathrm{max} \\
\cdot 0,2 \mathrm{MPa} \leq \sigma_{3} \leq 0,5 \mathrm{Mpa}\end{array}\end{array}$ & $\begin{array}{l}15,6 \\
16,4 \\
17,0\end{array}$ & & \\
\hline
\end{tabular}

Les études comparatives de Negussey et al. (1988) sur un sable d'Ottawa et Oyenuga et Tisot (1989) sur un sable de Moselle ont par ailleurs montré un bon accord entre $\varnothing_{\mathrm{c}}$ et l'angle $\varnothing_{\mathrm{cuc}}$ mesuré au point de contraction maximale dans un essai triaxial. L'utilisation de cette méthode indirecte conduit ici à $\varnothing_{\mathrm{cv}}=33^{\circ}$, soit $\tan \varnothing_{\mathrm{pv}}=$ 0,65 . Cette valeur s'inscrit bien dans la plage des valeurs généralement admises pour des grains siliceux.

\section{9}

\section{Mise en évidence du rôle temporaire de la cohésion}

Les figures 3 et 4 illustrent l'évolution de la résistance au cisaillement exprimée sous la forme $\tau / \sigma$ (en ordonnée à gauche) et du soulèvement moyen du boitier supérieur (en ordonnée à droite) avec le déplacement U. La figure 3 est donnée pour une série d'essais en place à Saint-Vallier et la figure 4 pour les essais conduits en laboratoire avec $\mathrm{d}_{\text {ax }}=50 \mathrm{~mm}$. Pour alléger la représentation, la relation V-U est donnée uniquement pour les plus faibles et plus fortes valeurs de $\sigma$.

Les positions $\mathrm{U}_{\text {pic }}$ de la contrainte maximale et $\mathrm{U}_{\text {, de }}$ la contrainte résiduelle, ainsi que les valeurs $V_{\max } d u$ soulèvement maximal et $\mathrm{dV} / \mathrm{dU}$ du gradient de soulèvement maximal sont précisées pour l'un des essais.

La comparaison des figures 3 et 4 indique que le remaniement et l'écrêtage simple à $50 \mathrm{~mm}$ n'altèrent pas le comportement pic-palier et le caractère dilatant des alluvions du Rhône en place.

La diminution du rapport $\tau / \sigma$ quand $\sigma$ augmente, qui est sensible dès le début de l'essai et très nette au voisinage du pic, prouve la manifestation d'une cohésion tant pour le sol en place que pour le sol reconstitué. Au-delà du pic, pour un déplacement qui peut varier entre 20 et $30 \mathrm{~mm}$ selon les conditions d'essai, les relations $\tau / \sigma_{\mathrm{n}}-U$ se confondent, ce qui démontre que la cohésion est détruite bien avant l'obtention de $\mathrm{V}_{\operatorname{mux}}$ et du palier.

\section{3}

\section{Effet de la dilatance}

L'analyse globale des résultats indique que la phase initiale de la contractance est d'autant plus accentuée que $\sigma_{0}$ est fort, que la position du gradient maximal $\mathrm{dV} / \mathrm{dU}_{\max }$ coincide avec celle du pic $\tau_{\operatorname{ma}} / \sigma_{\text {et }}$ et que $\mathrm{dV} / \mathrm{dU}_{\max }^{\max }$ et $\mathrm{V}_{\max }$ diminuent quand $\sigma_{0}$ augmente. Ces constatations sont en accord avec les connaissances sur la dilatance.

Le soulèvement optimal n'excède pas $6 \mathrm{~mm}$ et semble peu influence par la taille des grains. Par ailleurs l'obtention de $V_{\text {max }}$ est parfois concomitante à l'apparition du palier résiduel (voir Fig. 4), mais il arrive qu'elle le précède (voir Fíg. 3).

Pour des déplacements excédant $50 \mathrm{~mm}$ des aléas expérimentaux peuvent conduire à une altération du comportement du matériau: non planéité du sol support, rencontre de gros éléments dans le plan de cisaillement et désalignement du système de traction. Cette difficulté à maintenir le bon guidage du boîtier supérieur, surtout pour les essais en place, conduit à un basculement par l'avant du dispositif et à une diminution du soulèvement moyen. La relation V - U se radou- 


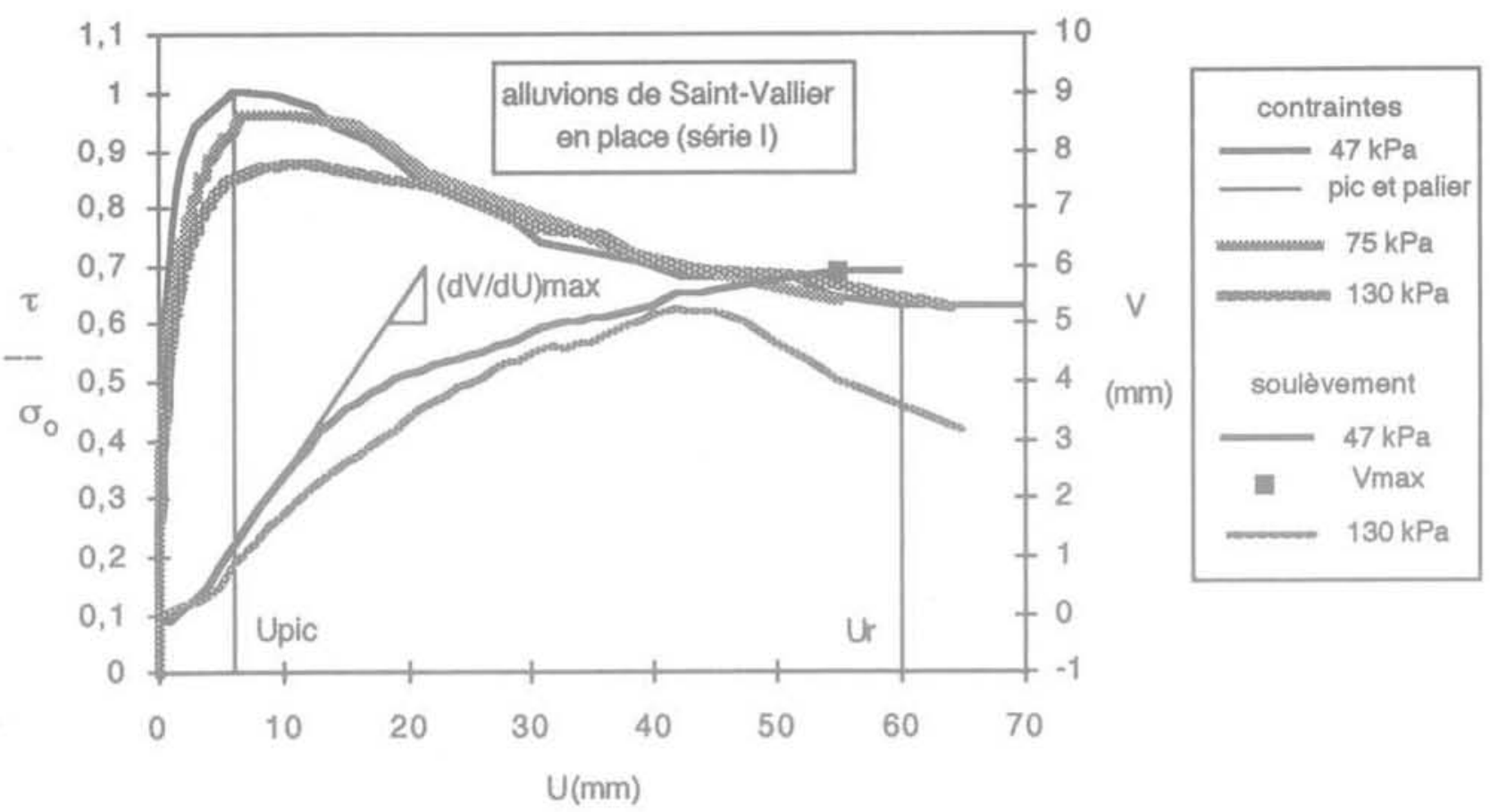

FG. 3 Exemple de relations contrainte - déplacement et soulèvement - déplacement dans un essai de cisaillement direct en place avec les alluvions du Rhône (Saint-Vallier, essai 1).

Example for shear stress versus displacement and vertical displacement versus displacement relationships in in situ direct shear test on Rhône alluvium materials (Saint-Vallier, test 1).

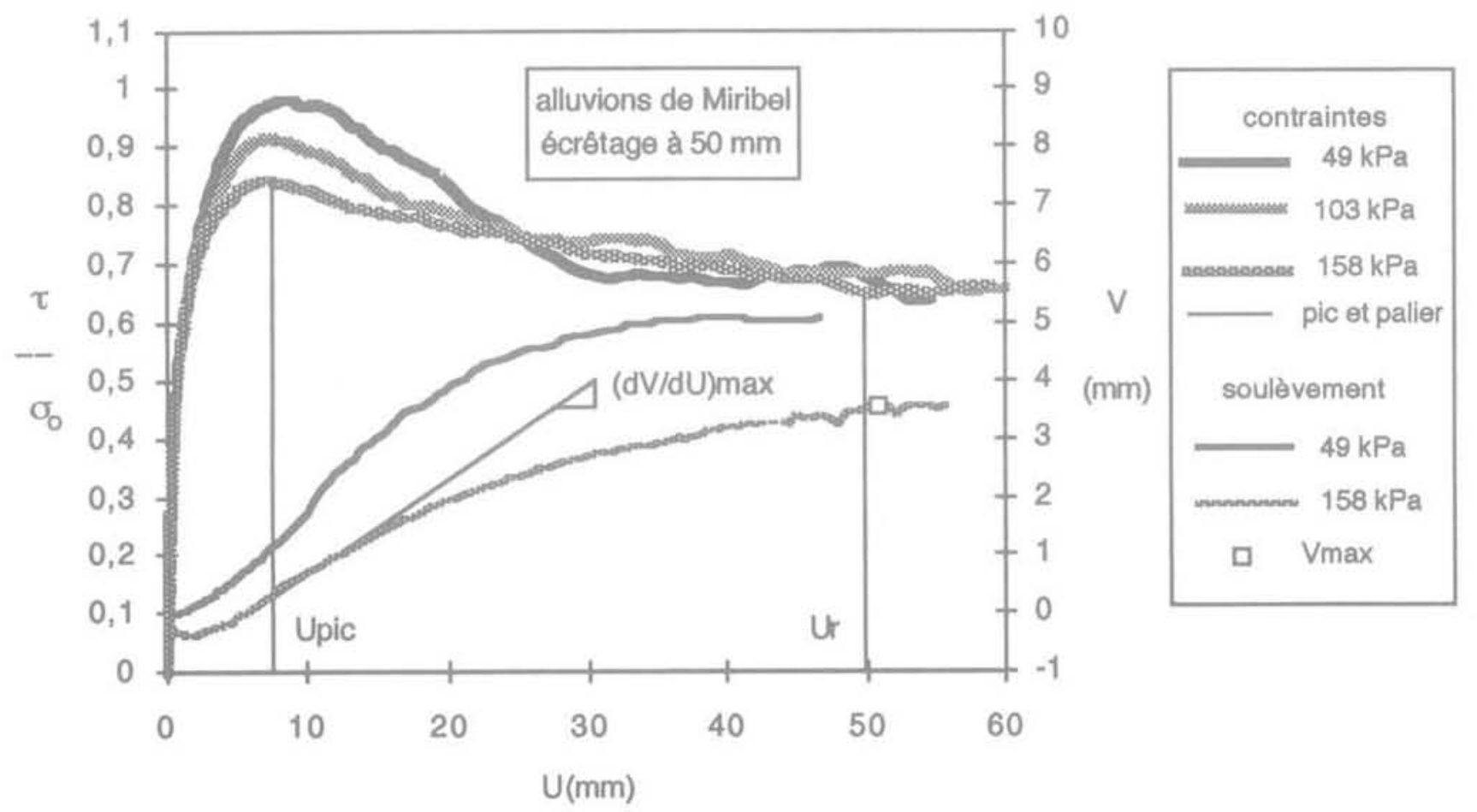

FIG.4 Exemple de relations contraintes - déplacement et soulèvement-déplacement dans un essai de cisaillement direct au laboratoire avec les alluvions du Rhône $\left(\mathrm{d}_{\mathrm{ma}}=50 \mathrm{~mm}\right)$.

Example of shear stress versus displacement and vertical displacement versus displacement relationships in laboratory direct shear test on Rhône alluvium materials ( $\mathrm{d}_{\max }=500 \mathrm{~mm}$ ). 


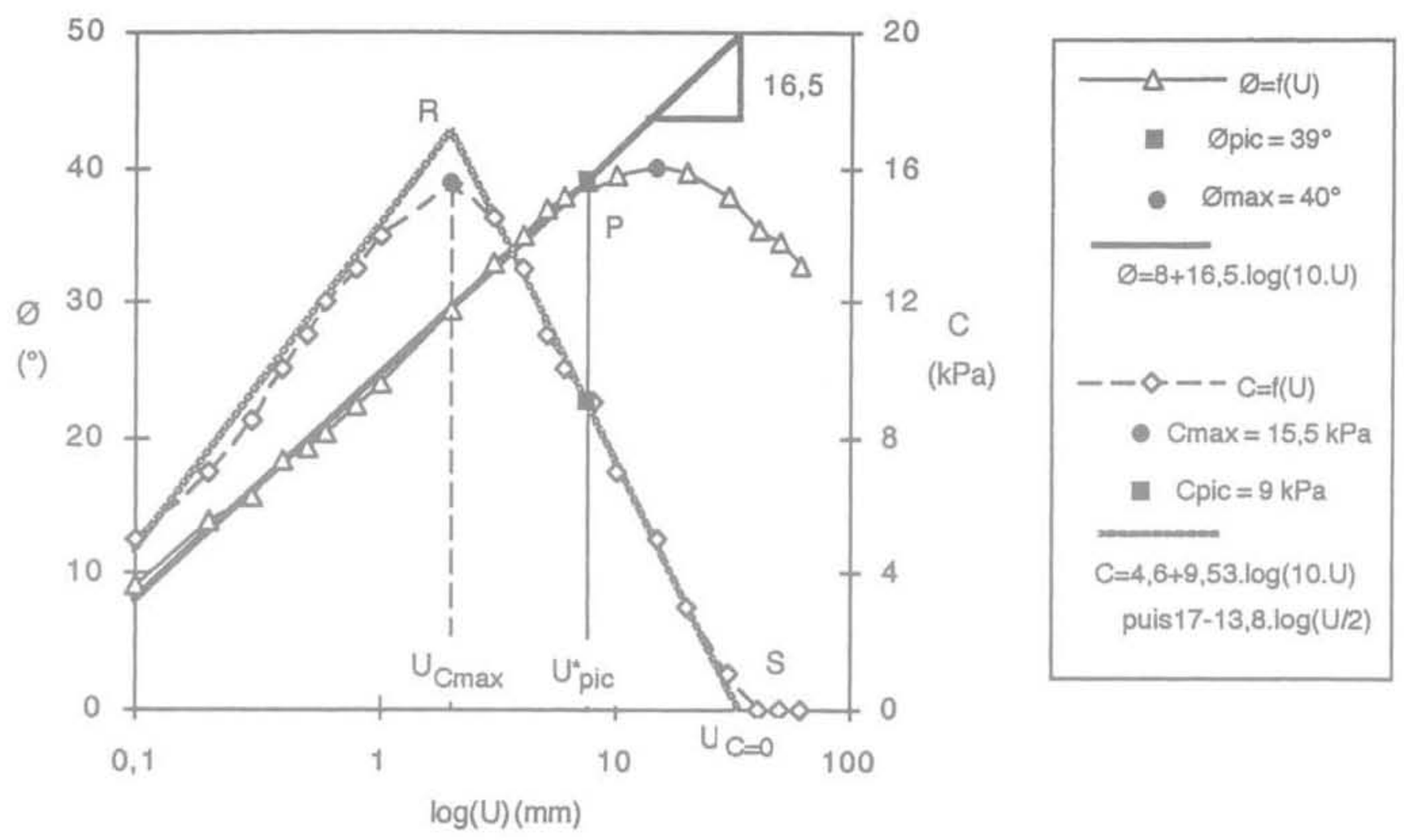

FG.5 Exemple de relations angle de frottement interne - déplacement et cohésion-déplacement (Saint-Vallier, essai 1).

Example of mobilized internal friction angle versus displacement and cohesion versus displacement relationships (Saint-Vallier, test 1).

cit alors au-delà de la valeur optimale $V_{\max }$ (voir Fig. 3, pour $\sigma_{0}=130 \mathrm{kPa}$ ).

\section{5}

\section{Mécanisme de la mobilisation de la résistance au cisaillement}

\section{1}

\section{Évolution de $\varnothing$ et $C$ avec le déplacement}

La description du mécanisme de progression de l'angle de frottement $\emptyset$ et de la cohésion $C$ avec le déplacement oblige à distinguer les valeurs optimales $\varnothing_{\max }$ et $C_{\text {mux }}$ qui peuvent être atteintes séparément par le tracé à Úgal des relations $\tau-\sigma$ et les valeurs $\varnothing$ et $C_{\text {pic }}$ déterminées conventionnellement avec les efforts au pic, dans le repère $\tau_{\max }-\sigma_{0}$.

Pour l'exemple du site de Saint-Vallier (Fig. 5), les évolutions de $\varnothing$ (en ordonnée à gauche) et de C (ordonnée à droite) sont schématisées par des relations linéaires dans un repère semi-logarithmique. Le positionnement de $\varnothing$ et $C_{\text {pr }}$ sur ce schéma correspond à un déplacement apparent $\mathrm{U}^{*}$ pic qui marque la fin de la progression linéaire de $\varnothing$. Pour cet exemple, la valeur $\varnothing_{\max }=40^{\circ}$ est supérieure à la valeur conventionnelle $\varnothing_{\text {pic }}=39^{\circ}$.

$$
\varnothing=\left(1,08 \varnothing_{\text {pic }}-32\right)+16,5 \log (10 \mathrm{U})
$$

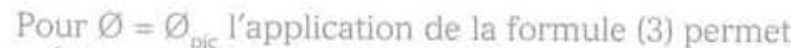
de préciser son domaine de validité.

$$
\begin{array}{ll} 
& U(\mathrm{~mm}) \leq U^{*}{ }_{\text {pic }}=8,7 \exp \left(-0,011 \varnothing_{\text {pic }}\right) \\
\text { soit } & U(\mathrm{~mm}) \leq 5,6 \mathrm{~mm} \text { pour } \emptyset_{\text {pic }}=40^{\circ} \\
& \mathrm{U}(\mathrm{mm}) \leq 6,3 \mathrm{~mm} \text { pour } \varnothing_{\text {pic }}=30^{\circ}
\end{array}
$$

Pour une gamme de valeurs courantes de $\varnothing_{\text {pu }}$ l'application numérique montre que le déplacement apparent au pic augmente modérément quand $\varnothing_{\text {pic }}$ diminue.

L'évolution de la cohésion avec log(U) est approchée par une relation bilinéaire dont la première partie conduit peu après la fin de la phase de contractance à une valeur optimale $\mathrm{C}_{\text {pax }}$, pour un déplacement $\mathrm{U}_{\mathrm{Cm}}$ inférieur à $\mathrm{U}^{*}{ }_{\text {pic }}$. Au-dela de $\mathrm{U}_{\mathrm{Cmax}}$ la décroissance de C est très rapide, et le matériau devient purement frottant alors que la dégradation de $\varnothing$ est juste amorcée. L'observation d'une valeur optimale de la cohésion précédant le pic pour des matériaux grossiers est également citée par Ortigosa (1989).

La difficulté à obtenir, comme pour $\varnothing$, une loi explicite $\mathrm{C}=\mathrm{f}(\mathrm{U})$ oblige à procéder par corrélations (voir $\$ 5$ 2 et $5-3$ ).

\section{2}

\section{Lois empiriques pour l'évolution conjointe de $C$ et $\varnothing$}

La figure 6 traduit l'évolution conjointe de la cohésion et de l'angle de frottement pour quelques séries 


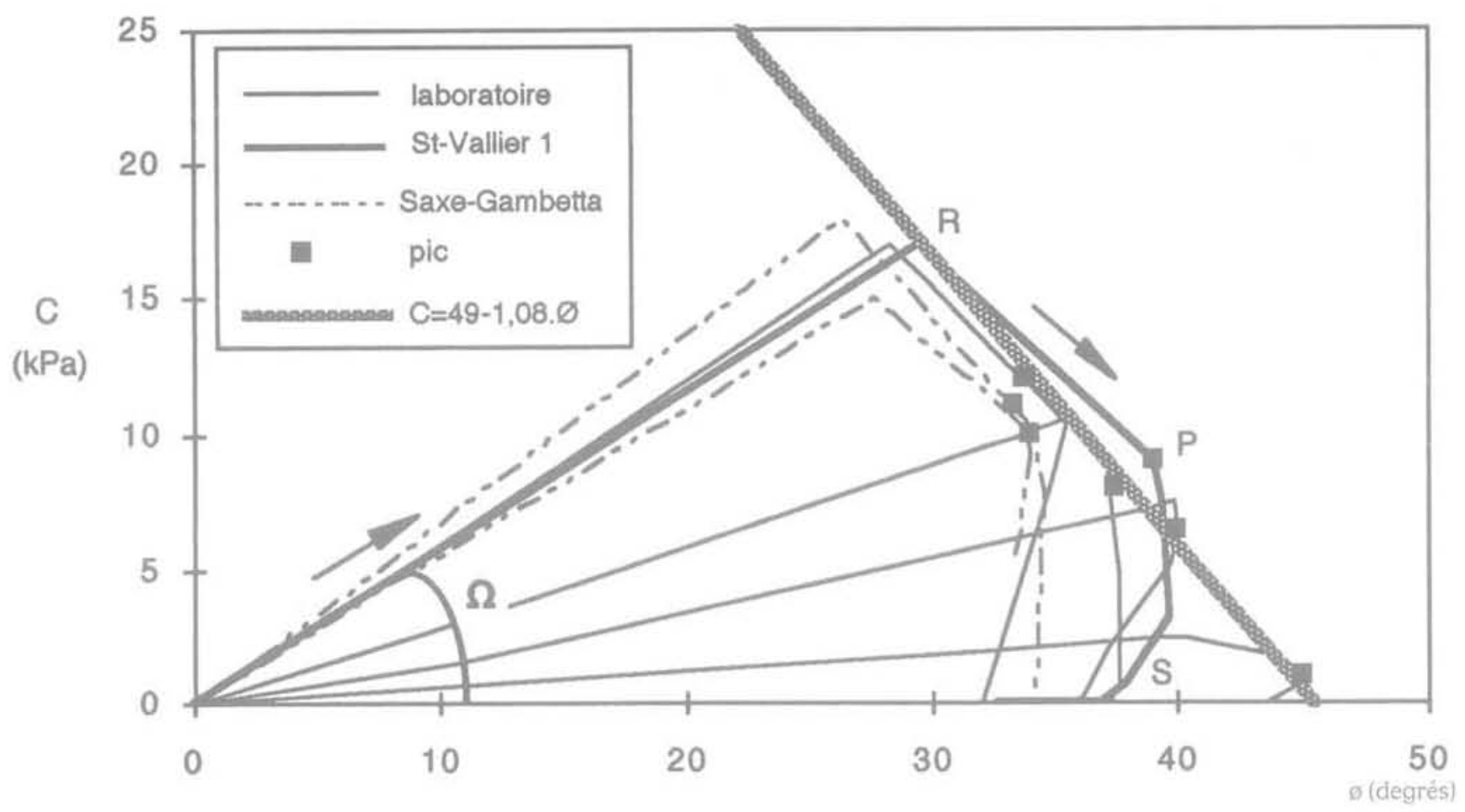

FG.6 Évolution conjointe de la cohésion avec I'angle de frottement. Mobilized cohesion versus mobilized internal friction angle.

d'essais citées aux tableaux I et II. La position des pics est donnée en symboles noirs. Pour l'exemple de SaintVallier (courbe ORPS en trait gras) la première partie OR représente l'augmentation conjointe de $\mathrm{C}$ et $\varnothing$ jusqu'au point $R$ de cohésion optimale $C_{\text {mix }}$. Cette phase linéaire de pente $\Omega$ satisfait à la relation (5).

$$
\mathrm{C}=\Omega \varnothing
$$

pour $\mathrm{U} \leq \mathrm{U}_{\text {Cmax }}$

Malgré la dispersion des points de mesure pour les essais en place dans la représentation $\Omega-F$, la figure 7 montre que la pente $\Omega$ est plus faible pour les essais de laboratoire, ce qui signifie que la reconstitution du matériau et le délai de quelques heures autorisé entre l'application de la charge et le cisaillement ne permettent pas de retrouver complètement la cohésion naturelle en place. A facteur d'amélioration égal, les expressions (6a) pour le sol en place et (6b) pour l'échantillon reconstitué indiquent que la cohésion est détruite pour moitié avec le remaniement.

$$
\begin{aligned}
& \Omega(\mathrm{kPa})=7,12-10 \mathrm{~F}_{\mathrm{a}} \\
& \Omega(\mathrm{kPa})=3,56-5 \mathrm{~F}_{\mathrm{a}}
\end{aligned}
$$

La seconde partie RP (Fig. 6) précise à la fois la diminution progressive de la cohésion et la poursuite de la mobilisation du frottement interne jusqu'au point P de résistance au cisaillement maximale. Les profils RP s'appuient sur une même enveloppe, représentée en grisé, dont l'approximation linéaire (7) convient pour le sol en place ou reconstitué.

$$
C(\mathrm{kPa})=49-1,08 \varnothing \text { (degré) }
$$

pour $\mathrm{U}_{\mathrm{Cmax}} \leq \mathrm{U} \leq \mathrm{U}_{\text {pic }}^{*}$

Pour décrire l'évolution de la cohésion avec le déplacement il suffit alors de combiner dans un premier temps les formules (3) et (5), en tenant compte de (6a) ou (6b), puis les expressions (3) et (7). Ensuite, comme présenté à la figure 5 , une extrapolation linéaire conduite entre $\mathrm{U}_{\text {Cmax }_{\max }}$ qui est déduit de l'égalité entre (5) et (7) et $U^{*}$ pic qui est tiré de (4) permet de poursuivre la démarche jusqu'au déplacement $\mathrm{U}_{c=0^{*}}$.

\section{3}

\section{Corrélation entre valeurs au pic et caractéristiques physiques}

L'examen de la figure 6 montre que l'expression (7) relative à l'enveloppe des tracés $C-\varnothing$ peut être étendue pour les valeurs au pic à la corrélation (8).

$$
C_{\text {pic }}(\mathrm{kPa})=49-1,08 \varnothing_{\text {pic }} \text { (degré) }
$$

La procédure d'évaluation de $\varnothing$ et $C$ avec $U$ (voir $\$ 5-1$ et 5-2) et la détermination de $C_{\text {pic }}$ avec (8) nécessi-

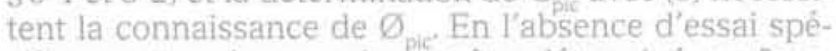
cifique cette valeur au pic peut être déterminée en fonction du facteur d'amélioration $\mathrm{F}_{\mathrm{a}^{\prime}}$ avec l'expression (9) tirée de la figure 8.

$$
\tan \varnothing_{\text {pic }}=3,7 \mathrm{~F}_{\mathrm{z}}-1,62
$$

Pour $\mathrm{C}_{\text {pic }}=0$ les relations générales (1), (2), (6a), (6b), (8) et (9) concourent aux valeurs limites $\varnothing_{\text {Cpic }=0}^{\max }=45^{\circ} 4$ de l'angle de frottement au pic et $F_{{ }^{\max }=0}=0,712$ du facteur d'amélioration. L'examen des valeurs $\gamma_{d}$ et $\mathrm{Cu}$ mesurées en place (tableau I) montre que l'obtention de la condition $\left[\mathrm{F}_{\mathrm{a} \mathrm{C}_{\text {pic }}=0}=\varnothing_{\mathrm{C}_{\text {pic }}=0}^{\max }\right]$ relève d'une conjoncture exceptionnelle. Ainsi, pour $\mathrm{d}_{\max }=31,5 \mathrm{~mm}$, le couple de valeurs $\left[\varnothing_{\text {pic }}=45^{\circ} ; C_{\text {pic }}=1 \mathrm{kPa}\right.$ ] figurant au tableau IV n'a pu être obtenu qu'au prix d'un compactage très énergique conduisant à $\gamma_{d}=24,4 \mathrm{kN} / \mathrm{m}^{3}$ (Tableau II). 


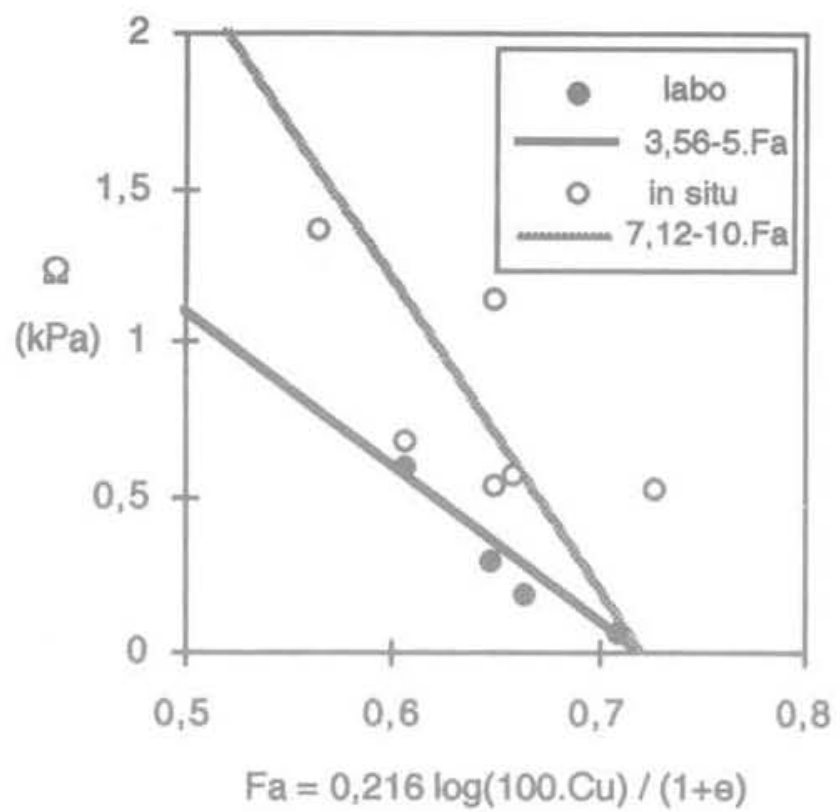

FIG, 7 Corrélation entre l'angle et le facteur $\Omega$ d'amélioration $\mathrm{F}$.

Relation between $\Omega$ and coefficient $F_{n}$

\section{4}

\section{Interprétation globale}

Dans les tableaux récapitulatifs III et IV, $\varnothing_{v_{\operatorname{mmx}}}$ représente l'angle mesuré au point de soulèvement maximal et $\mathrm{U}^{*}$ pic correspond à la simulation propre à chacun des essais comme présenté à la figure 5 . Cette valeur peut s'écarter légèrement du résultat déduit de la corréla-

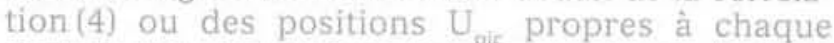
contrainte $\sigma_{0}$. Par ailleurs, hormis pour le site de Lyon Saxe-Gambetta (III), l'angle $\varnothing_{\operatorname{mux}}$ est supérieur à $\varnothing_{\text {pic }}$ : l'écart n'excède cependant pas $2^{\circ}$, ce qui est logique compte tenu de la faible influence de $\sigma_{0}$ sur $U_{p}$ (voir Fig. 3 et 4).

Pour compléter les tendances marquantes tirées des relations (1) à (9), les valeurs conventionnelles $\emptyset_{\text {pir }} \varnothing_{\text {. }}$ et $\varnothing_{c v}$ de l'angle de frottement doivent être comparées entre elles et situées par rapport aux valeurs $\varnothing_{\max }$ et $\emptyset_{v_{\max }}$ déduites des relations $\tau-\sigma_{0}$ à $\mathrm{U}$ égal. La figure 9 , qui traduit la correspondance entre les angles de frottement mesurés au pic et au palier, conduit ainsi à la relation linéaire (10).

$$
\tan \varnothing_{f}=0,48+0,2 \tan \varnothing_{p i c}
$$

Cette expression prouve que l'exploitation classique d'un essai de cisaillement direct peut conduire à un angle de frottement au pic inférieur à la fois à l'angle de frottement résiduel et à l'angle de frottement critique. Ce cas de figure survient ici pour $\varnothing_{\text {pir }}<31^{\circ}$, ou pour $\mathrm{C}_{p j c}>15,5 \mathrm{kPa}$ d'après la relation (8). Par ailleurs, bien qu'alors supérieur à $\varnothing_{\text {, }} \varnothing_{\text {pic }}$ reste encore inférieur à $\varnothing_{c y}$ pour $C_{p l c}>13,4 \mathrm{kPa}$.

Dans la méthode d'exploitation qui est présentée, la triple condition $\varnothing_{\max } \geq \varnothing_{\mathrm{y}_{\max }} \geq \varnothing_{\mathrm{cy}} \geq \varnothing_{\mathrm{r}}$ est vérifiée à l'exception du site de Vénissieux et l'écart $\varnothing_{\mathrm{ymax}}-\varnothing_{\mathrm{cv}}$ est inférieur à $1^{\circ}$ à l'exception du site de Saint-Vallier (série 1).

Il ressort de cette analyse que l'état critique des alluvions du Rhône peut être approché avec un excès infé-

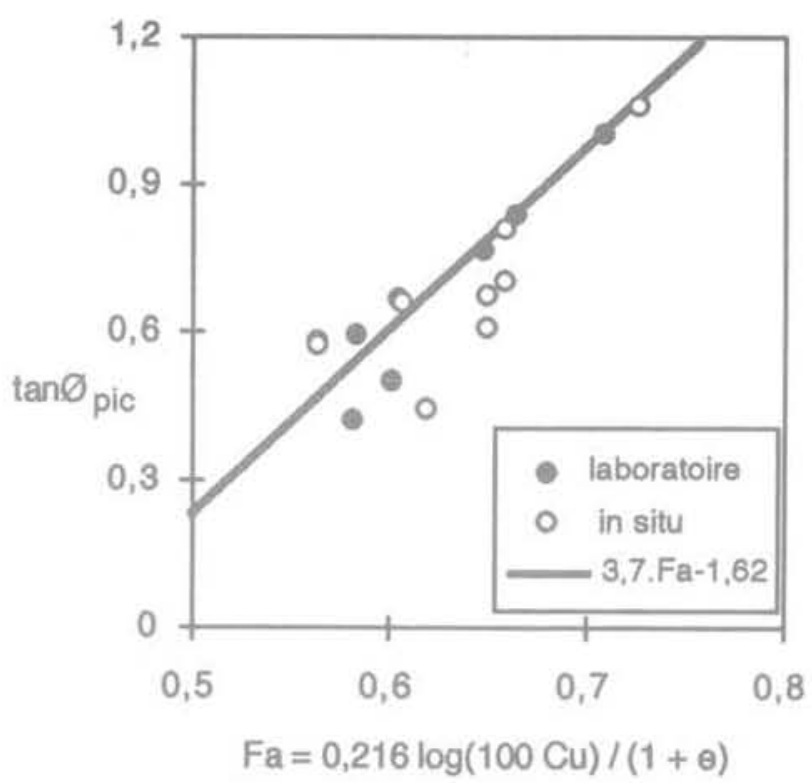

FG. 8 Corrélation entre l'angle de frottement au pic et le facteur d'amélioration $\mathrm{F}_{\mathrm{a}}$. Relation between ultimate friction angle and coefficient $F_{\text {s. }}$

rieur à 1 degré au point de soulèvement optímal du boîtier supérieur dans un essai de cisaillement direct à disposition symétrique. La difficulté de poursuivre l'essai avec une valeur nulle du gradient $\mathrm{dV} / \mathrm{dU}$ explique que l'angle de frottement chute ensuite vers la valeur $\varnothing_{r}<$ $\emptyset_{V_{\max }}$

L'étude phénoménologique de la mobilisation de la résistance au cisaillement des alluvions du Rhône peut donc être schématisée par une succession de phases dont l'ordre chronologique est décrit à la figure 10. La zone hachurée correspond au domaine d'évolution possible de $\varnothing_{\text {pic }}$ et $C_{\text {pic }}$.

\section{6}

\section{Étude de la dilatance}

\section{1}

\section{Analyse de la dilatance dans un essai de cisaillement direct}

Le modèle de la dilatance «en lame de scie» (sawtooth) présenté à la figure 11 a été utilisé par Bolton (1986), puis repris par Jewell (1989), Houlsby (1991), Creed (1994), Ogunro (1996) pour définir l'angle de dilatance $\psi$ et les deux lois d'écoulement d'un sol granulaire purement frottant dans un essai de cisaillement direct. La première loi décrit l'évolution conjointe des paramètres de dilatance et de résistance au cisaillement au cours de l'essai et la deuxième loi corrèle ces paramètres mesurés au pic.

Dans ce modèle, l'angle de frottement $\varnothing$ mesuré dans le plan de cisaillement imposé $\mathrm{x}$ - $\mathrm{x}^{\prime}$ est décomposé selon un angle de frottement $\varnothing_{c^{\prime}}$ qui est mobilisé le long des microfacettes de glissement préférentiel orientées suivant une direction $\mathrm{z}-\mathrm{z}^{\prime}$, et un angle de dilatance $\psi$ qui correspond à l'orientation de ces microfa- 
TABLEAU III Caractéristiques de cisaillement des alluvions du Rhône (essais in situ). Shear parameters of Rhône alluvium materials (in situ tests).

\begin{tabular}{|c|c|c|c|c|c|c|c|c|}
\hline Essais in situ & $\mathrm{C}_{\mathrm{K}}$ & $\begin{array}{l}\mathrm{U}_{\mathrm{Cmin}} \\
\text { (min) }\end{array}$ & $\theta_{\text {Dig }}$ & $\mathrm{C}_{(\mathrm{kPa})}$ & $\begin{array}{l}U^{\circ} \\
\text { (mm) }\end{array}$ & $\theta \theta_{\max }$ & $\varnothing_{U_{\max }}$ & (C) \\
\hline $\begin{array}{l}\text { Lyon Part-Dieu (I) } \\
\text { Lyon Part-Dieu (II) } \\
\text { Lyon Part-Dieu (III) } \\
\text { Lyon Part-Dieu (IV) } \\
\text { Lyon Saxe-Gambetta (I) } \\
\text { Lyon Saxe-Gambetta (II) } \\
\text { Lyon Saxe-Gambetta (III) } \\
\text { Lyon Garibaldi } \\
\text { Vénissieux } \\
\text { Saint-Vallier (I) } \\
\text { Saint-Vallier (II) } \\
\text { Saint-Vallier (III) } \\
\text { Saint-Vallier (IV) } \\
\text { Millery } \\
\text { Feyzin }\end{array}$ & $\begin{array}{l}- \\
- \\
- \\
\overline{18} \\
\overline{1} \\
15 \\
\overline{3} \\
\frac{1}{17} \\
27 \\
- \\
- \\
20 \\
-\end{array}$ & $\begin{array}{l}- \\
- \\
- \\
- \\
1,7 \\
\overline{1,8} \\
- \\
4 \\
2 \\
2,4 \\
- \\
- \\
- \\
-\end{array}$ & $\begin{array}{l}37^{\circ} 5 \\
31^{\circ} 5 \\
26^{\circ} 2 \\
33^{\circ} 5 \\
33^{\circ} 3 \\
30^{\circ} 0 \\
34^{\circ} 0 \\
33^{\circ} 0 \\
29^{\circ} 8 \\
39^{\circ} 0 \\
31^{\circ} 3 \\
34^{\circ} 8 \\
23^{\circ} 8 \\
46^{\circ} 6 \\
30^{\circ} 2\end{array}$ & $\begin{array}{c}3 \\
8 \\
13 \\
7 \\
11 \\
16 \\
10 \\
20 \\
20 \\
9 \\
16 \\
8,5 \\
16 \\
3 \\
16\end{array}$ & $\begin{array}{c}- \\
- \\
- \\
- \\
4,5 \\
- \\
4,3 \\
- \\
9,7 \\
7,6 \\
7 \\
- \\
- \\
9,5 \\
-\end{array}$ & $\begin{array}{c}- \\
- \\
- \\
- \\
34^{\circ} 6 \\
- \\
33^{\circ} 4 \\
- \\
30^{\circ} 5 \\
40^{\circ} 0 \\
33^{\circ} 4 \\
- \\
- \\
46^{\circ} 9 \\
-\end{array}$ & $\begin{array}{l}- \\
- \\
- \\
- \\
- \\
- \\
- \\
- \\
- \\
35^{\circ} 0 \\
33^{\circ} 3 \\
- \\
- \\
- \\
-\end{array}$ & $\begin{array}{l}- \\
- \\
- \\
25^{\circ} 5 \\
30^{\circ} 8 \\
29^{\circ} 6 \\
31^{\circ} 2 \\
30^{\circ} 7 \\
32^{\circ} 7 \\
31^{\circ} 0 \\
30^{\circ} 0 \\
29^{\circ} 2 \\
34^{\circ} 5 \\
30^{\circ} 6\end{array}$ \\
\hline
\end{tabular}

TABLEAUN Caractéristiques de cisaillement des alluvions du Rhône (essais de laboratoire). Shear parameters of Rhône alluvium materials (laboratory tests).

\begin{tabular}{|c|c|c|c|c|c|c|c|c|}
\hline Essais de laboratoire & $\mathrm{C}_{(\mathrm{kPa})}$ & $\begin{array}{l}\mathrm{U}_{\text {Ginim }} \\
\text { (mim) }\end{array}$ & $\theta_{\text {pic }}$ & $\frac{\mathrm{C}_{\text {pili }}}{(\mathrm{kPa})}$ & $\begin{array}{l}U^{2} \\
\text { (mm) }\end{array}$ & $\emptyset_{\max }$ & $\theta_{v_{\max }}$ & $\varphi_{1}$ \\
\hline $\begin{array}{l}\text { Miribel } 80 \mathrm{~mm} \\
\text { Miribel } 50 \mathrm{~mm} \\
\text { Miribel } 31,5 \mathrm{~mm} \text { (I) } \\
\text { Miribel } 31.5 \mathrm{~mm} \text { (II) } \\
\text { Miribel } 20 \mathrm{~mm} \text { (I) } \\
\text { Miribel } 20 \mathrm{~mm} \text { (II) } \\
\text { Vénissieux } 31,5 \mathrm{~mm} \\
\text { Satolas } 20 \mathrm{~mm}\end{array}$ & $\begin{array}{l}10,5 \\
7,5 \\
2.5 \\
- \\
17 \\
- \\
- \\
-\end{array}$ & $\begin{array}{l}4 \\
6 \\
3 \\
- \\
2,6 \\
- \\
- \\
-\end{array}$ & $\begin{array}{l}37^{\circ} 5 \\
40^{\circ} 0 \\
45^{\circ} 0 \\
26^{\circ} 5 \\
23^{\circ} 0 \\
33^{\circ} 7 \\
30^{\circ} 7 \\
26^{\circ} 1\end{array}$ & $\begin{array}{l}8 \\
6,5 \\
1 \\
17 \\
18 \\
12 \\
12 \\
13\end{array}$ & $\begin{array}{l}5,4 \\
6,2 \\
5,7 \\
- \\
- \\
5,5 \\
- \\
-\end{array}$ & $\begin{array}{l}37^{\circ} 6 \\
40^{\circ} 0 \\
45^{\circ} 0 \\
- \\
- \\
35^{\circ} 3 \\
33^{\circ} 0 \\
-\end{array}$ & $\begin{array}{c}33^{\circ} 0 \\
33^{\circ} 0 \\
33^{\circ} 8 \\
- \\
33^{\circ} 4 \\
34^{\circ} 0 \\
- \\
-\end{array}$ & $\begin{array}{l}33^{\circ} 4 \\
33^{\circ} 2 \\
34^{\circ} 3 \\
30^{\circ} 1 \\
31^{\circ} 4 \\
31^{\circ} 8 \\
30^{\circ} 9 \\
-\end{array}$ \\
\hline
\end{tabular}

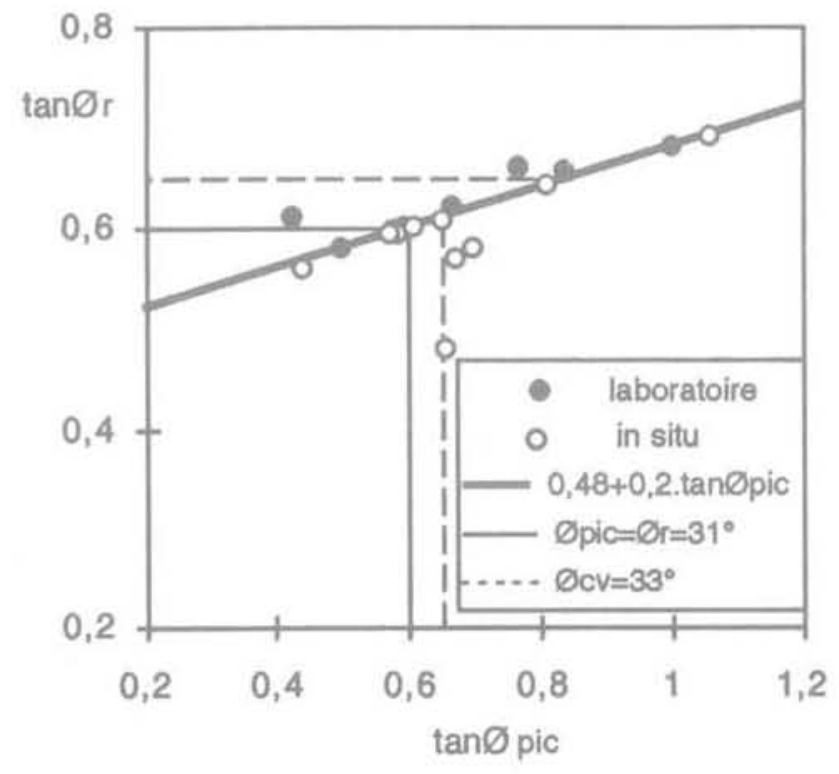

FG. 9 Corrélation entre l'angle de frottement résiduel et l'angle de frottement au pic. Residual friction angle versus ultimate friction angle.

cettes. La loi d'écoulement associée à ce modèle correspond à l'écriture (11).

$$
\varnothing=\varnothing_{c}+\psi
$$

La loi d'écoulement (11) présente cependant l'inconvénient de ne pas faire apparaitre de relation explicite entre $\varnothing$ et $\psi$, l'angle $\varnothing_{c}$ pouvant varier entre $\varnothing_{c y}$ et l'angle de frottement interparticules $\emptyset_{\text {u }}$ selon les conditions d'essai (Fig. 12). Les propositions de lois faisant intervenir la valeur critique $\varnothing$ sont bien résumées par Jewell (1989). L'expression(12) proposée par Stroud (1971) pour l'essai de cisaillement simple peut être approchée avec un excès de l'ordre de $10 \%$ par un essai de cisaillement direct conforme à la disposition symétrique. L'angle de dilatance est alors calculé selon (13) à partir du gradient de soulèvement dV/dU du boîtier supérieur et $\varnothing$ est donné par (14).

$$
\begin{aligned}
& \tan \varnothing=\sin \varnothing_{\mathrm{cv}}+\tan \psi \\
& \tan \psi=\mathrm{dV} / \mathrm{dU} \\
& \tan \varnothing=\tau / \sigma_{\mathrm{o}}
\end{aligned}
$$

\section{2}

\section{Résultats expérimentaux}

Le calcul du gradient $\mathrm{dV} / \mathrm{dU}$ nécessite le lissage préalable de la relation $\mathrm{V}-\mathrm{U}$ avec une fonction polynomiale vérifiant d'une part la condition $\mathrm{dV} / \mathrm{dU}=0$ aux points de contractance maximale et de soulèvement maximal et présentant d'autre part un point d'inflexion concomitant à l'effort de pic. Les lois (13) et (14) n'étant par ailleurs applicables qu'à un sol purement frottant, c'est-à-dire pour $\mathrm{U}>\mathrm{U}_{\mathrm{c}}=0$ avec les alluvions du Rhône, la figure 13 relative à l'exemple des alluvions écrêtées à 


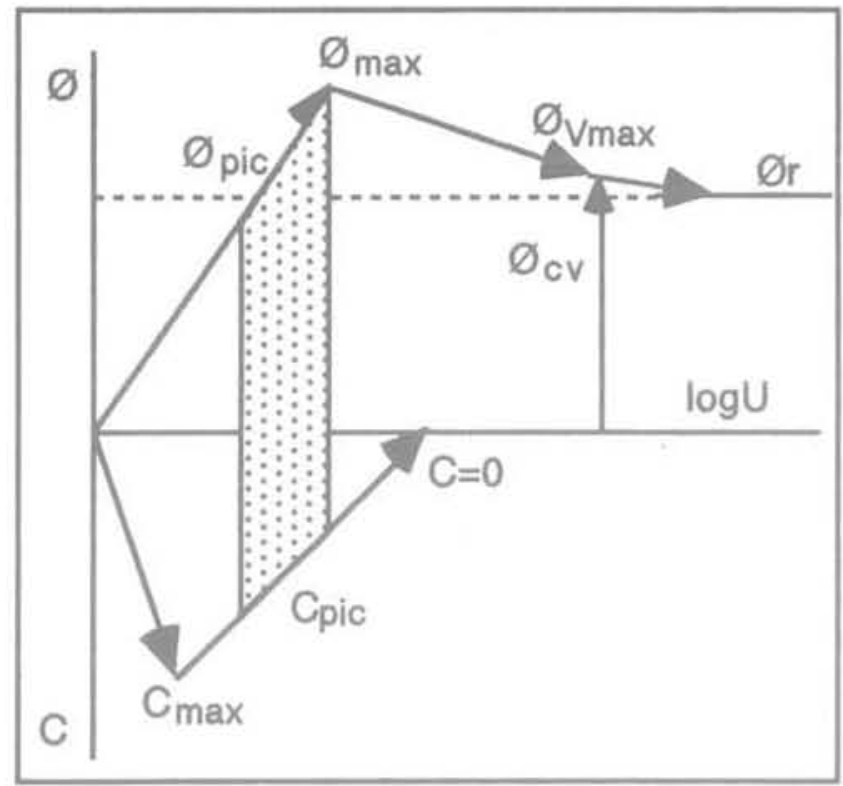

FIG:10 Schématisation de l'évolution des paramètres de cisaillement avec le déplacement.

Schematic model of components of shear strength as function of displacement.
$50 \mathrm{~mm}$ est donnée sous la forme plus générale $\tau / \sigma_{0}-$ $\mathrm{dV} / \mathrm{dU}$,

Dans le domaine des valeurs $\mathrm{dV} / \mathrm{dU}$ positives la loi d'écoulement présente deux parties distinctes MP et PN, qui se chevauchent, de part et d'autre du point de rebroussement $\mathrm{P}$ marquant le passage concomitant de l'effort de pic et du gradient optimal dV/dU . Pour des valeurs $\tau / \sigma$ et $\mathrm{dV} / \mathrm{dU}$ croissantes, la phase MP représente le passage du point de contractance maximale à l'effort de pic (point P). Pour des valeurs $\tau / \sigma$ et $d V / d U$ décroissantes, la $2^{c}$ phase PN correspond au radoucissement de l'effort jusqu'au point de soulèvement $V_{\text {mix }}$.

\section{3}

\section{Lois d'écoulement}

\section{pour les alluvions du Rhône}

Malgré certaines difficultés de lissage de la courbe $\mathrm{V}$-U, les phases MP et PN de la loi d'écoulement peuvent le plus souvent être approximées par des relations linéaires distinctes, de pentes respectives $m$ et $n$ (Fig. 14). Pour la première partie, qui satisfait à l'expression (15), l'ordonnée à l'origine $\tau_{\mathrm{M}} / \sigma_{\mathrm{O}}$ croît et $\mathrm{m}$ décroit quand $\sigma_{\mathrm{o}}$ augmente. $\tau_{\mathrm{M}} / \sigma_{\mathrm{o}}$ varie globalement entre 0,44 et 0,69 et $m$, qui fait l'objet de la corrélation (16), peut varier entre 0,9 et 2. Cette analyse ne permet pas de préciser l'apport de la disposition symétrique adoptée au laboratoire vis-à-vis du chargement libre de l'essai en place, ni d'apprécier l'influence de l'écrêtage.

$$
\frac{\tau}{\sigma_{0}}=\frac{\tau_{\mathrm{M}}}{\tau_{\mathrm{o}}}+\mathrm{m}\left(\frac{\mathrm{dV}}{\mathrm{dU}}\right)
$$

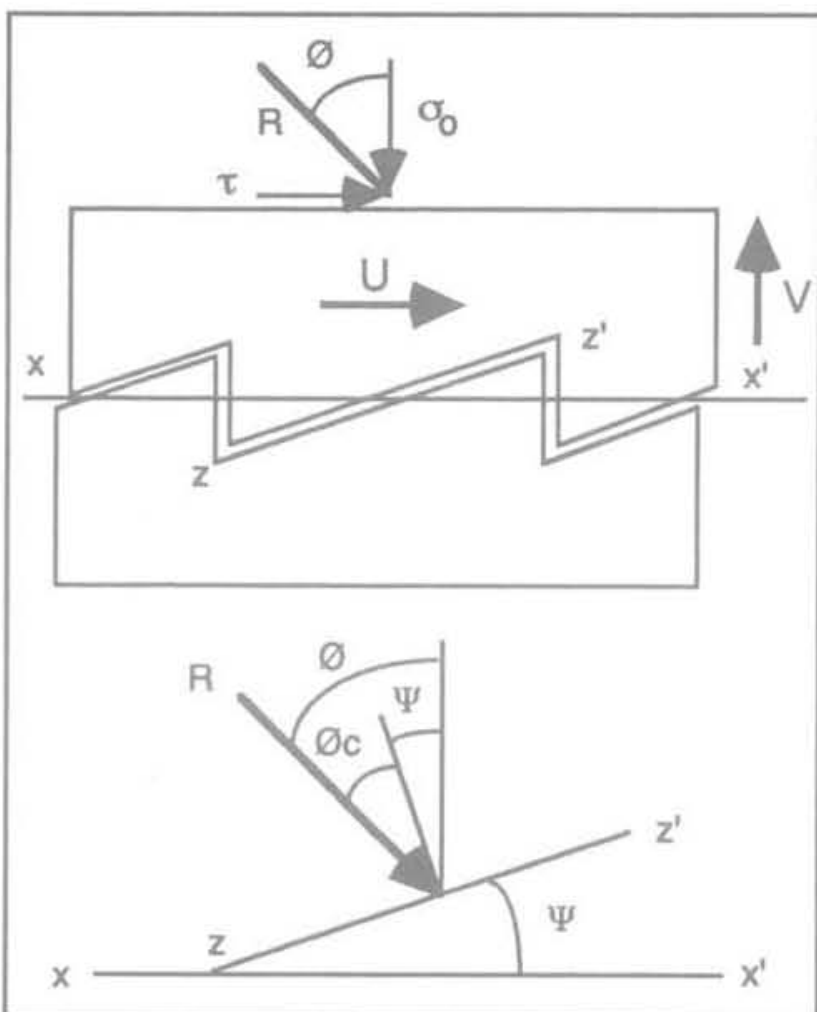

FIG.11 Le modèle de dilatance d'après Bolton (1986).

The sawtooth model for dilatancy (Bolton. 1986).

$$
\mathrm{m}=\frac{\tan \varnothing_{\text {pic }}\left(1-\frac{34}{\sigma_{0}}\right)+\frac{34}{\sigma_{0}}-\frac{\tau_{\mathrm{M}}}{\tau_{0}}}{\tan \varnothing_{\text {pic }}\left(1-\frac{34}{\sigma_{0}}\right)+\frac{34}{\sigma_{0}}-0,65}
$$

pour $\mathrm{U} \leq \mathrm{U}^{*}{ }_{\text {pic }}$

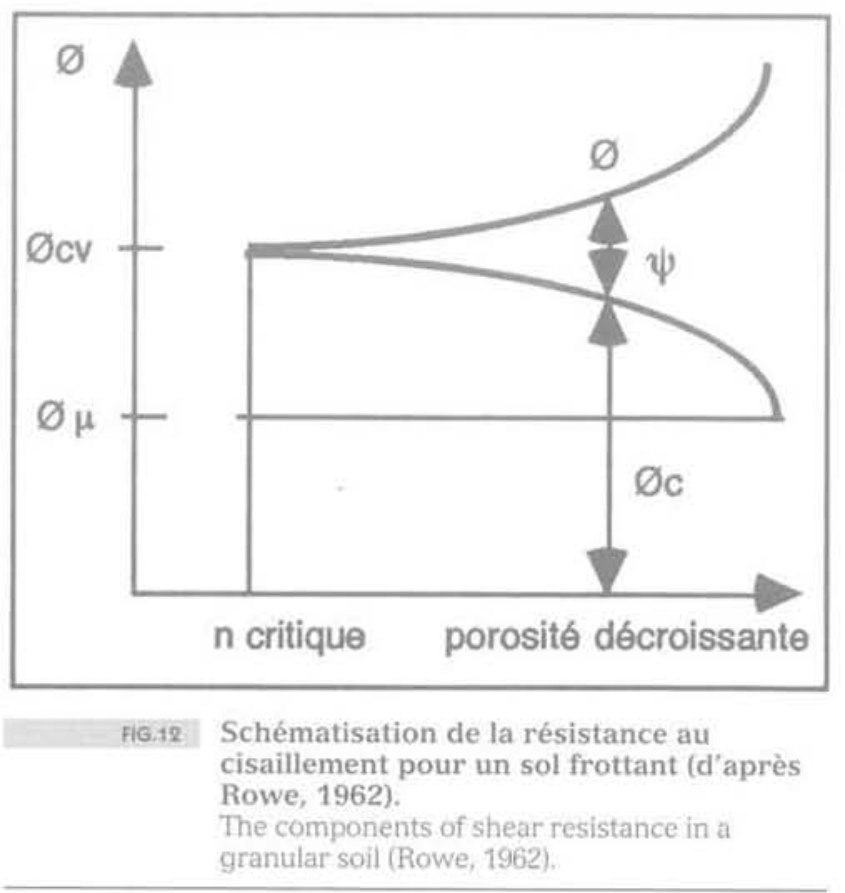




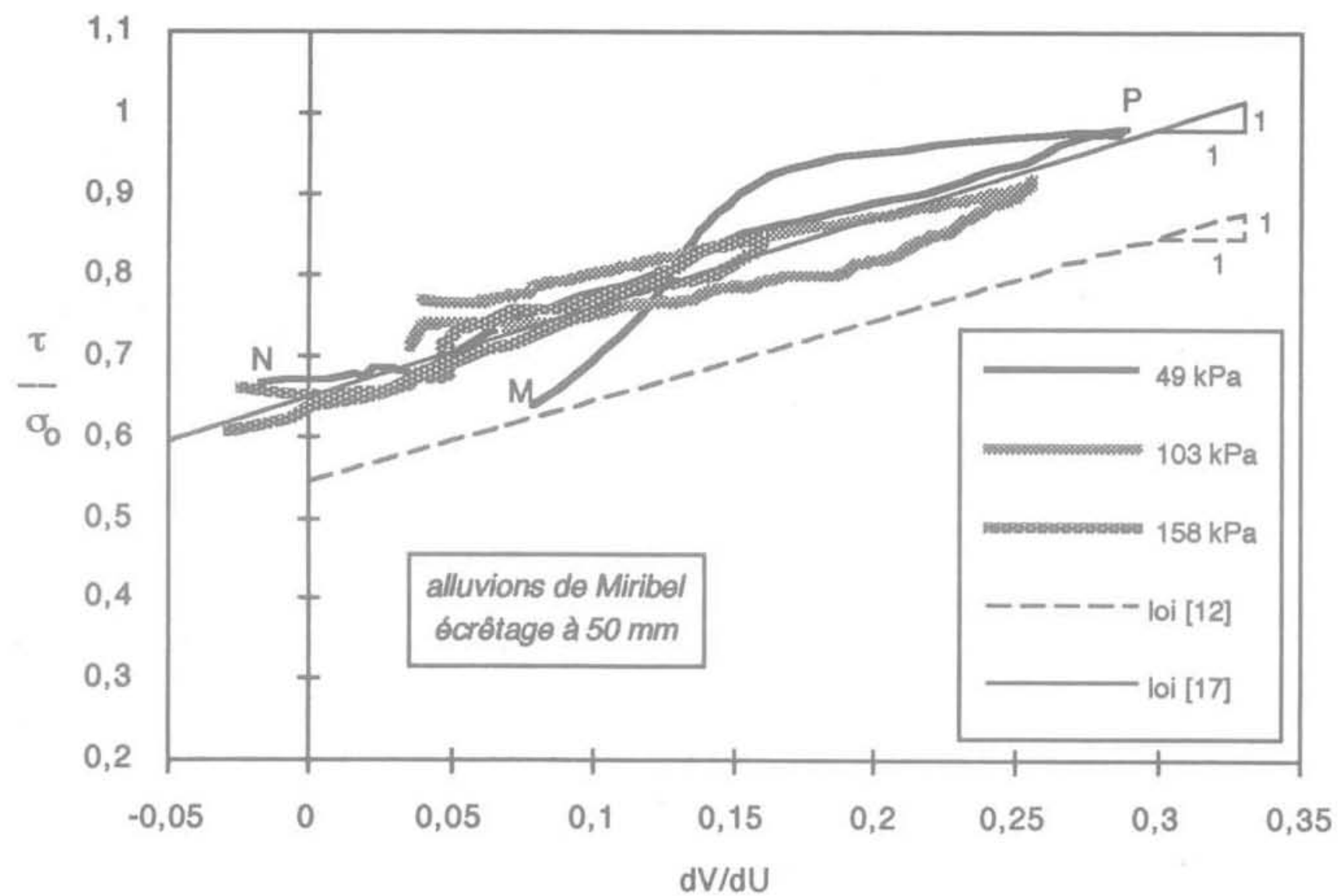

F16.13 Exemple de loi d'écoulement contrainte de cisaillement - gradient de soulèvement dans un essai de cisaillement direct avec les alluvions du Rhône $\left(\mathrm{d}_{\max }=50 \mathrm{~mm}\right)$.

Example of shear stress versus rate of dilation flow rule during direct shear test on Rhône alluvium materials.

La pente et l'ordonnée à l'origine de la deuxième partie sont indépendantes des conditions d'essai. La valeur $n=1,06$ proposée par Ogunro (1996) pour les essais de laboratoire a été abaissée à $n=1$ pour un meilleur calage avec les essais in situ et l'ordonnée $\tau_{1} / \sigma_{\text {. }}$ $=\tan \emptyset_{\mathrm{cv}}=0,65$ de l'expression réduite (17) est en accord avec le principe que l'angle de frottement critique soit approché avec un léger excès au point de soulèvement maximal, pour $\mathrm{dVdU}=0$.

$$
\frac{\tau}{\sigma_{0}}=\tan \varnothing_{\mathrm{cv}}+\left(\frac{\mathrm{dV}}{\mathrm{dU}}\right)
$$

pour $\mathrm{U} \geq \mathrm{U}^{*}{ }_{\text {pic }}^{*}$

L'écart observé entre les lois d'écoulement empiriques (16) et (17) et la loi (12) se situe dans le fuseau d'épaisseur $\tan \varnothing_{c \mathrm{v}}-\sin \varnothing_{\mathrm{cv}}=0,105$ représenté à la figure 13. Il peut être attribué d'une part à l'empêchement du développement de la dilatance par la cohésion et expliqué d'autre part par l'analyse de Jewell (1989) (voir \$6-1).

La loi d'écoulement (18) obtenue au pic avec les

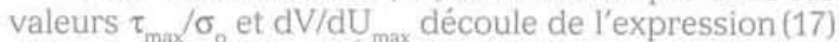
relative à la deuxième phase PN. La figure 15 indique qu'elle est indépendante du mode de réalisation de l'essai.

$$
\frac{\tau_{\max }}{\sigma_{\mathrm{o}}}=\tan \emptyset_{\mathrm{cv}}+\left(\frac{\mathrm{dV}}{\mathrm{dU}}\right)_{\max }
$$

\section{4}

\section{Correspondance entre la dilatance et le comportement pic-palier}

La relation entre la dilatance et le radoucissement de l'effort dans l'essai de cisaillement direct est établie à partir de la définition $\Delta \tau=\left(\tau_{\max }-\tau_{\mathrm{r}}\right)$ donnée à la figure 16, en supposant que les paramètres $C_{p j e}, \varnothing_{\text {pic }}$ et

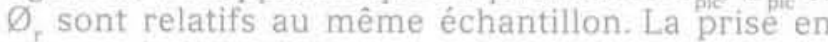
compte de (10) et (18) conduit à l'écriture (19) entre le gradient $\mathrm{dV} / \mathrm{dU} U_{\max }$ et l'écart relatif $\Delta \tau / \sigma_{i}$.

$$
\begin{aligned}
& \frac{\Delta \tau}{\sigma_{0}}=\frac{\tau_{\operatorname{mix}}}{\sigma_{0}}-\frac{\tau_{\mathrm{r}}}{\sigma_{0}}=\frac{\tau_{\max }}{\sigma_{0}}-\tan \varnothing_{\mathrm{T}} \\
& \frac{\Delta \tau}{\sigma_{n}}=\frac{\tau_{\max }}{\sigma_{n}}-0,48-0,2\left(\frac{\tau_{\max }}{\sigma_{n}}-\frac{\mathrm{C}_{\text {pic }}}{\sigma_{\mathrm{n}}}\right) \quad \text { d'après (10 } \\
& \frac{\Delta \tau}{\sigma_{0}}=0,8\left[0,65+\left(\frac{\mathrm{dV}}{\mathrm{dU}}\right)_{\max }\right]-0,48+0,2 \frac{\mathrm{C}_{\text {pic }}}{\sigma_{0}} \text { d'après } \\
& \quad\left(\frac{\mathrm{dV}}{\mathrm{dU}}\right)_{\max }=1,25 \frac{\Delta \tau}{\sigma_{0}}-0,05-0,25 \frac{\mathrm{C}_{\text {pic }}}{\sigma_{0}}
\end{aligned}
$$

La figure 17 confirme que la loi linéaire $\mathrm{dV} / \mathrm{dU}_{\max }=$ $1,25 \Delta \tau / \sigma_{-}-0,05$ constitue la borne supérieure de la relation $\mathrm{dV} / \mathrm{dU}_{\max }-\Delta \tau / \sigma_{0}$ pour le cisaillement direct des 


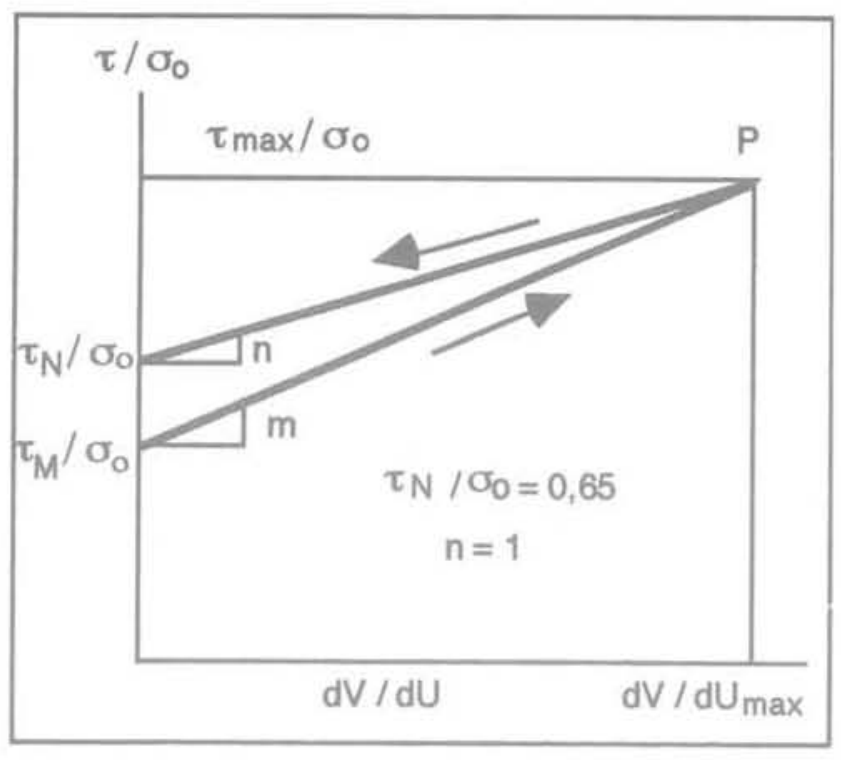

FIG.14 Schématisation de la loi d'écoulement. Schematic model of flow rule,

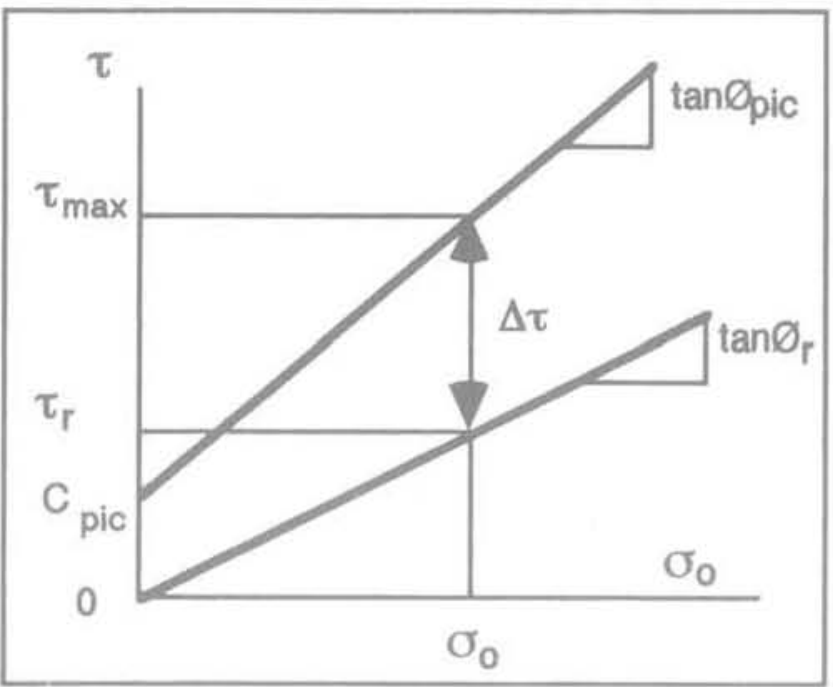

FIG.16 Définition du radoucissement $\Delta \tau$. Definition of straim softening $\Delta \tau$. alluvions du Rhône. Elle correspond au sol supposé purement frottant et montre le lien étroit entre le développement de la dilatance et le radoucissement postpic de l'effort. L'effet de la cohésion sur ce comportement tient à l'écart $0,25 \mathrm{C}_{\text {pic }} / \sigma_{0}$ et devient négligeable pour $\sigma_{0}$ $\geq 200 \mathrm{kPa}$ compte temu des valeurs courantes de la cohésion au pic (voir Tableaux III et IV).

\section{5}

\section{Effet de l'écrêtage non compensé}

Les points de mesure qui correspondent sur les figures 15 et 17 à un comportement pic-palier et à un

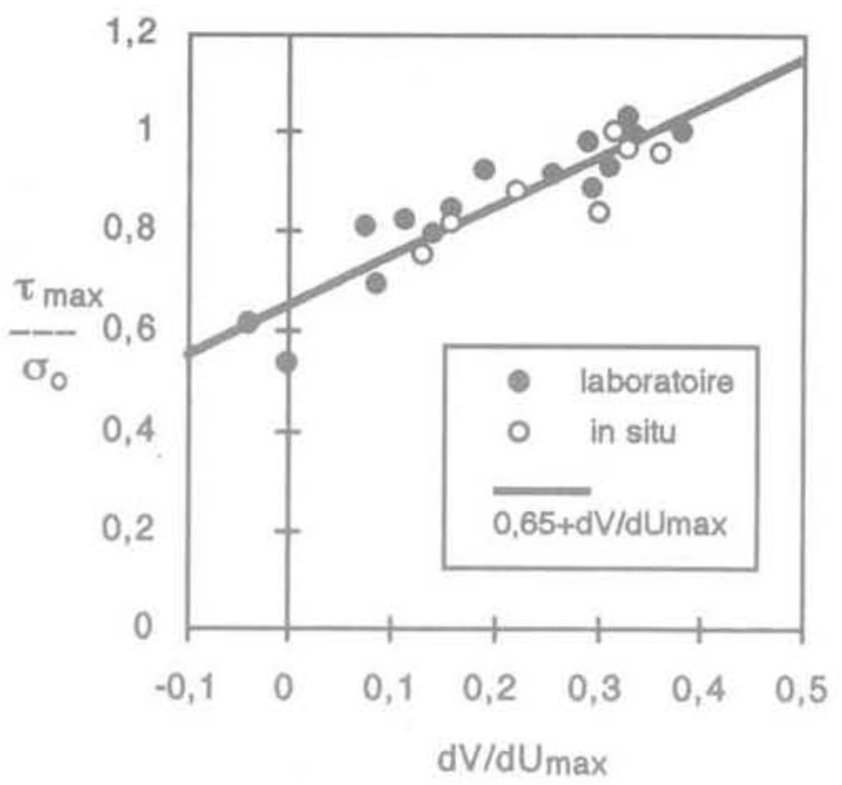

FiG,15 Loi d'écoulement au pic. Ultimate flow rule.

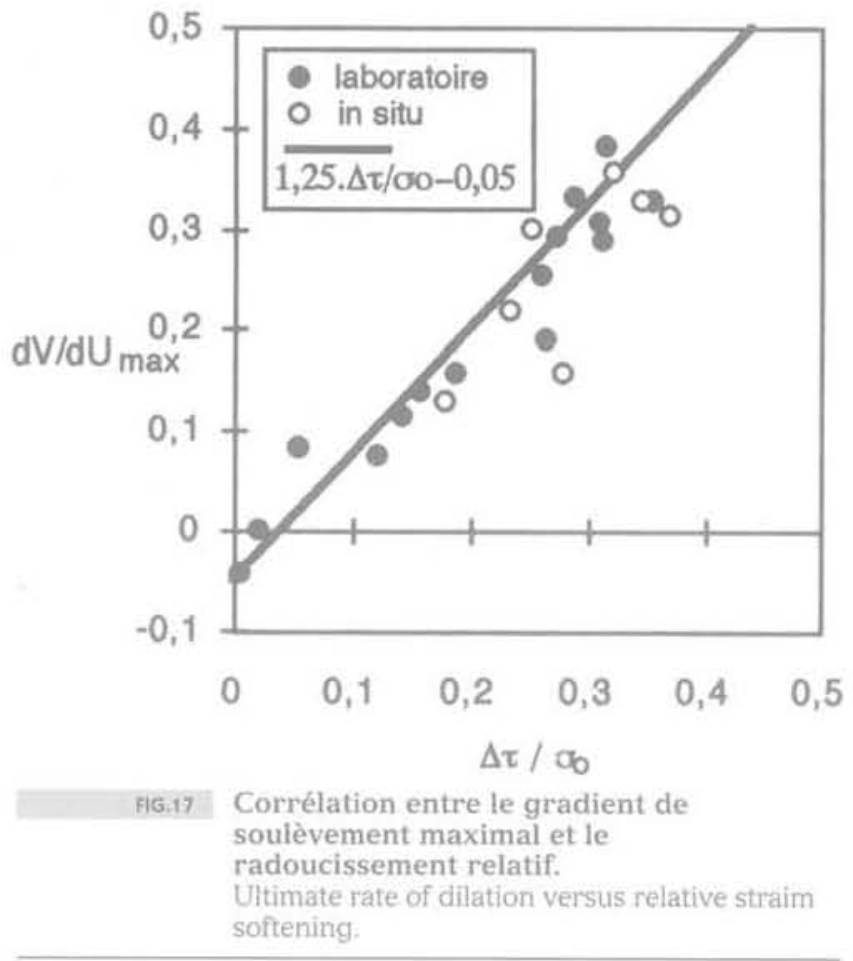

échantillons de laboratoire écrêtés à $20 \mathrm{~mm}$ et soumis aux plus fortes valeurs de $\sigma_{\sigma}$, ce qui prouve les limites d'un écrètage non compensé sur la conservation du comportement du matériau. La figure 1 révèle en effet qu'un écrêtage simple à $20 \mathrm{~mm}$ conduit à un rejet d'éléments parfois supérieur à $25 \%$, alors que plusieurs auteurs admettent que le dépassement de cette tolérance doit être accompagné d'une compensation ou d'un remodelage par similitude (Post, 1979; Dendani, 1988).

L'analyse des résultats ayant par ailleurs prouvé que les alluvions du Rhône conservent toutes leurs propriétés pour $\mathrm{d}_{\max } \geq 31,5 \mathrm{~mm}$, avec un rejet inférieur à $25 \%$, la règle susmentionnée apparaît donc vérifiée ici. 


\section{Conclusion}

La procédure décrite dans cet article permet de préciser les paramètres de cisaillement des alluvions du Rhône à partir de lois de comportement empiriques de formulation simple, complémentaires, qui peuvent être classées en deux groupes:

- les lois du premier groupe permettent de corréler entre eux ou avec le facteur d'amélioration $\mathrm{F}$ défini par (2) les paramètres conventionnels établis aủ pic ou au palier. Le couplage des lois (8) et (9) avec (2) est ainsi d'un grand intérêt pratique, car il permet d'estimer les valeurs au pic à partir de propriétés physiques et granulométriques du matériau facilement identifiables; le projeteur aura cependant intérêt à effectuer des recoupements avec d'autres sources d'information. Par ailleurs, la loi d'écoulement établie au pic permet la prise en compte de la dilatance dans les ouvrages, ou peut encore être utilisée pour établir des corrélations avec les caractéristiques pressiométriques;

- en décrivant l'évolution de $\varnothing, C$ et de la dilatance avec le déplacement imposé, les lois (3) à (7) et (15) à (17) du deuxième groupe peuvent être d'un grand intérêt pour le calcul en service de structures déformables, pour l'étude de la stabilité de pentes où la gradation des paramètres de cisaillement est sensible le long de la surface de glissement, et pour les ouvrages oú la dégradation des paramètres de cisaillement est manifeste.

Dans certaines de ces lois empiriques la prise en compte de l'atténuation de la cohésion avec la reconstitution du matériau mérite une étude plus approfondie pour définir notamment l'influence du facteur temps sur le phénomène.

\section{Bibliographie}

Bacot J. - Contribution à l'étude du frottement entre une inclusion souple et un matériau pulvérulent: cas de la Terre Armée. Thèse Sci., INSA Lyon, 1981, $389 \mathrm{p}$.

Bolton M.D. - The strength and dilatancy of sands. Géotechnique, vol. $36, \mathrm{n}^{*} 1$, 1986, p. 65-78.

Bourdeau Y., Laréal P., Marchal I. - Résistance au cisaillement des alluvions du Rhône, Proc.XII ICSMFE, Rio de Janeiro, vol. 1, août 1989, p. 695-696.

Cambefort H. - Curiosités des massifs alluvionnaires et des nappes d'eau. Supplément aux Annales de ITTBTP, $n^{\circ} 211-212$. juillet-août 1965, p. 923-947.

Creed M.J. - A laboratory introduction to critical state soil mechanics, Proc. XIII ICSMFE, New Delhi, vol.4, janvier 1994, p. $1479-1482$.

Dendani H. - Comportement de matêriaux de barrages en terre: étude expérimentale et modélisation, Thèse de Doctorat, INP Grenoble, 1988, 291 p.

David L - Formations glaciaires et fluvioglaciaires de la région Lyonnaise, Doc. Labo, Géologie Fac. Sci. Lyon, n²2, 1967, $159 \mathrm{p}$.

Guichard J.-C. - La résistance au cisaillement des sols grossiers : étude des allu- vions du Rhône, DEA Génie Civil, INSA Lyon, 1995, 135 p.

Houlsby G.T. - How the dilatancy of soils affects their behaviour, Proc. X ECSMFE, Florence, vol. 4, mai 1991. p. $1189-1202$.

Jewell R.A. - Direct shear tests on sand, Géotechnique, vol. 39, n², 1989, p. 309322.

Kastner R. - Excavations profondes en site urbain: problèmes liès à la mise hors d'eau, dimensionnement des soutènements butonnès. Thèse Sci., INSA Lyon. 1982, $409 \mathrm{p}$.

Laréal P., Bourdeau Y., Lambert P., Cottereau $\mathrm{Cl}$. - Essais de cisaillement in sifu sur des sols alluvionnaires de la vallée du Rhōne, Travaux, $n=459-460$, juinjuillet 1973, p. 52-59.

Laréal P. - Sols de la région lyonnaise : prévision de leur comportement mécanique à l'aide d'essais in situ et en vraie grandeur. Thèse Sci., Univ. Claude-Bernard. Lyon, 1975, 253p.

Mogami T. - On the angle of internal friction of rockfill materials. VII ICSMFE, Mexico, Specialty session $n^{\circ} 13$, vol. E1: Contributions and discussions on mechanical properties of rockfill and gravel materials, août 1969, p. 25-42.
Negussey D., Wijewickreme W.K.D., Vaid Y.P. - Constant-volume friction angle of granular materials, Canadian Geotechnical Journal, vol. 25, 1988, p. 50-55.

Ogunro V.T. - Le comportement d'interface de géosynthétiques avec des sables et des sols grossiers: influence des conditions d'essais, Thèse de Doctorat, INSA Lyon, 1996, 281 p.

Ortigosa P. - Propriétés géotechniques des sols grossiers, Proc. XII ICSMFE, Rio de Janeiro, vol. 5, aoút 1989, p. 2881-2883.

Ovenuga D.A. Tisot J.-P. - Looking for an intrinsic key for sands. Proc. Int. Conf. on Micromechanics of Granular Media : Powders and grains, Clermont-Ferrand, septembre 1989, p. 171-175, Edited by Biarez and Gourves, Rotterdam, AA. Balkerna, 1989.

Post G. - Design parameters for fill, Pro. VII ECSMFE, Brighton, vol 4, 1979, p. 209-215,

Rowe P.W. - The stress-dilatancy relation for static equilibriun of an assembly of particles in contact, Proc. Royal Society of London, series A vol. 269, 1962, p. 500-527.

Stroud M.A. - The behaviour of sands at low stress levels in the simple shear apparatus, Ph. D. thesis, University of Cambridge, 1971. 